
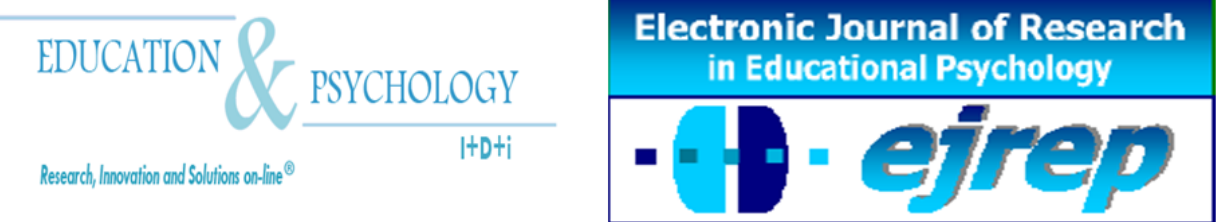

Editorial EOS

\title{
La estimación en el cálculo y en la medida: fundamentación curricular e investigaciones desarrolladas en el Departamento de Didáctica de la Matemática de la Universidad de Granada
}

\author{
Isidoro Segovia Alex \\ Enrique Castro Martínez
}

Departmento de Didáctica de la Matemática, Universidad de Granada

\section{España}

isegovia@ugr.es,ecastro@ugr.es

Dirección Postal: Isidoro Segovia. Facultad de Ciencias de la Educación. Departamento de Didáctica de la Matemática. Campus Cartuja, s/n, 18071 - Granada.

(C) Education \& Psychology I+D+i and Editorial EOS (Spain) 


\title{
Resumen
}

Investigadores del Departamento de Didáctica de la Matemática de la Universidad de Granada que forman parte del Grupo de Investigación "FQM193. Pensamiento Numérico" han estado trabajando desde 1985 en estimación, una de las componentes del pensamiento numérico. En este trabajo se hace una revisión global de las aportaciones más relevantes de este grupo de investigación en estimación; se comienza por una reflexión teórica y curricular, se hace una revisión de la literatura de investigación y se describen algunas de las investigaciones realizadas en el seno del grupo de investigación.

Palabras clave: Investigación en Estimación en Matemáticas, Pensamiento Numérico, Currículum

Recibido: 04/02/09 Aceptación inicial: 04/02/09 Aceptación final: 23/03/09

\begin{abstract}
Researchers of the Department of Mathematics Education at the University of Granada who are members of the research group "FQM193. Numerical Thinking", have been working since 1985 on one of the component of the number thinking, estimation. Our aim in this work is develop a global review of the most important contributions of our research group to estimation. Firstly, we perform some theoretical and curricular considerations. Secondly, awe present a revision of the research literature, and finally we conclude with a description of some of the researches that have been carried out in our research group.
\end{abstract}

Keywords: Research in Estimation in Mathematics, Number Thinking, Curriculum

Received: 02/04/09 Initial Acceptance: 02/04/09 Final Acceptance: 03/23/09 


\section{Introducción}

La estimación en cálculo y medida es una competencia matemática que se ha incorporado al currículo de matemáticas desde los años ochenta; dada su novedad, desde esa década y especialmente en Estados Unidos, se han realizado muchas investigaciones sobre el tema tratando de clarificar los elementos que caracterizan la estimación así como la forma de incluirla en el currículo. En el Departamento de Didáctica de la Matemática de la Universidad de Granada y dentro del grupo de investigación Pensamiento Numérico hemos trabajado en la temática desde una doble perspectiva: una reflexión curricular considerando los resultados de las investigaciones y que se presenta en la primera parte y un aporte de investigación en algunos aspectos que estaban por desarrollar.

\section{Aspectos teóricos de la Estimación}

\section{El concepto de Estimación}

El término estimación tiene múltiples usos y campos de aplicación. Es por tanto conveniente comenzar por precisar el concepto de estimación al cual se refiere este trabajo. La estimación es un "juicio sobre el valor del resultado de una operación numérica o de la medida de una cantidad, en función de circunstancias individuales del que lo emite" (Segovia, Castro, Rico y Castro, 1989, p.18) ${ }^{1}$. En el concepto anterior aparecen dos tipos de estimación:

a) Estimación en cálculo, referido a las operaciones aritméticas y a los juicios que pueden establecerse sobre sus resultados. Ejemplo: una estimación del resultado de 2345 multiplicado por 52 es 120000.

b) Estimación en medida, referido a los juicios que pueden establecerse sobre el valor de una determinada cantidad o bien la valoración que puede hacerse sobre el resultado de una medida. Dentro de la estimación en medida se distingue entre dos grupos de magnitudes: continuas y discretas. Por ejemplo, una estimación, para el caso de magnitudes continuas, es la valoración que hacemos sobre la estatura de una persona cuando la comparamos con la nuestra propia; para el caso de magnitudes discretas es la estimación del número de personas que asisten a una manifestación.

\footnotetext{
${ }^{1}$ El concepto de estimación dado, así como algunas de las consideraciones que en este trabajo se van a hacer están tomadas de los libros Estimación en Cálculo y Medida (Segovia, Castro, Rico y Castro, 1989) y Estimación de cantidades discretas. Estudio de variables y procesos (Segovia, 1997)
} 
El concepto general de estimación tiene implícitas las características dadas por Reys (1984) y completadas por Segovia, Castro, Rico y Castro (1989, p.21):

1. Consiste en valorar una cantidad o el resultado de una operación aritmética.

2. El sujeto que hace la valoración tiene alguna información, referencia o experiencia sobre la situación que debe enjuiciar.

3. La valoración se realiza por lo general de forma mental.

4. Se hace con rapidez y empleando números lo más sencillos posibles.

5. El valor asignado no es exacto, pero sí adecuado para tomar decisiones.

6. El valor asignado admite distintas aproximaciones dependiendo de quien realice la valoración.

\section{Estimación y Sentido Numérico}

El Sentido Numérico tiene una estrecha relación con la estimación. Con el ánimo de aclarar ideas respecto a su significado, en 1989 el consejo editorial de la revista Arithmetic Teacher, dedicó un número de esta revista a realizar una reflexión sobre el Sentido Numérico con dos objetivos, estimular una discusión sobre la definición de Sentido Numérico y dar ideas prácticas de cómo conseguir que los estudiantes desarrollen mejor su sentido numérico (Thompson y Rathmell, 1989). Para Edwars (1984) el Sentido Numérico es una forma de aritmética mental y una cierta capacidad de comparar números. Para el NCTM (1989) el desarrollo del Sentido Numérico implica,
a) comprender bien el significado de los números,
b) tener desarrolladas relaciones múltiples entre los números,
c) reconocer las magnitudes relativas de números,
d) conocer los efectos relativos de las operaciones con números,
e) desarrollar referentes para las medidas.

Para Sowder (1988), el Sentido Numérico es una red de conceptos bien organizada que permite relacionar números y propiedades de las operaciones y proporciona habilidad para trabajar con magnitudes numéricas. Greeno (1991) se refiere a importantes capacidades, como flexibilidad de cálculo mental, estimación numérica y juicio cuantitativo y emplea la metáfora del "ambiente" conceptual como modelo para su análisis y estudio; el campo conceptual es comparado con un ambiente en el que la gente aprende a vivir e interactuar. Lo define como "una competencia cognitiva que resulta de la actividad en un campo donde se aprende a interactuar sucesivamente con los variados recursos del campo, incluyendo conocer qué recursos 
La estimación en el cálculo y en la medida: fundamentación curricular e investigaciones desarrolladas en el Departamento de Didáctica de la Matemática de la Universidad de Granada

ofrece el ambiente, conocer cómo encontrar recursos y usarlos en las actividades, percibir y comprender patrones sutiles, resolver rutinariamente problemas ordinarios y generar nuevos conocimientos" (p.170).

En la línea de Greeno está la descripción de Sentido Numérico dada por Howden (1989): "El sentido numérico se puede describir como una buena intuición sobre números y sus relaciones. Se desarrolla gradualmente como resultado de explorar números, visualizarlos en contextos variados y relacionarlos con procedimientos que no se limiten a los algoritmos tradicionales" (p.11).

El Ministerio de Educación y Ciencia, MEC (2006), en el Real Decreto 1513/2006, de 7 de diciembre, por el que se establecen las enseñanzas mínimas de la Educación Primaria, el Sentido Numérico es definido como el "dominio reflexivo de las relaciones numéricas que se pueden expresar en capacidades como: habilidad para descomponer números de forma natural, comprender y utilizar la estructura del sistema de numeración decimal, utilizar las propiedades de las operaciones y las relaciones entre ellas para realizar mentalmente cálculos".

Aunque las opiniones acerca de qué es el Sentido Numérico sean diferentes, todas incluyen la Estimación como parte del mismo o lo consideran íntimamente interrelacionado.

\section{Estimación y Cálculo Mental}

En los procesos de estimación del resultado de una operación o de la medida de una cantidad, el cálculo mental tiene un papel muy destacado. No quiere decir esto que la estimación tenga como única herramienta de resolución los algoritmos mentales de cálculo; una estimación puede realizarse con algoritmos de lápiz y papel o calculadora; pero en la mayor parte de las situaciones, esto no es posible o deseable, y por tanto, se hace necesario el cálculo mental.

Reys (1984, p.548) establece dos características que distinguen al cálculo mental; la primera característica es que el cálculo mental produce respuestas exactas, mientras que la segunda, es que emplea procedimientos mentales de realización, sin ayuda externa de herramientas tales como el lápiz y el papel. No hay contradicción entre la producción de respuestas exactas, con los valores aproximados que produce la estimación; Reys hace referencia al cálculo mental, exclusivamente, sin relacionarlo con la estimación. Cuando el Cálculo Mental se utiliza en procedimientos de estimación, hay una selección previa de números sencillos con 
los que se opera mentalmente; es esta elección de números la que da lugar respuestas aproximadas. Da cinco razones para defender la enseñanza del cálculo mental en la escuela:

1) es un prerrequisito para el desarrollo de la aritmética escrita;

2) es un promotor del conocimiento de las estructuras de los números y sus propiedades;

3) es un promotor de la creatividad, del conocimiento independiente e incita a los estudiantes a tener ingenio con números grandes;

4) contribuye a la mejora en la resolución de problemas;

5) es una base para el desarrollo de técnicas de cálculo estimativo.

Gómez (1994, pp.35-45) desarrolla más ampliamente las razones que motivan la inclusión del cálculo mental en el currículum para la enseñanza obligatoria, entre las cuales se encuentra también la relación de éste con la estimación. Así, una buena base para la estimación consiste en lograr una destreza adecuada sobre algoritmos mentales que sean fáciles de memorizar y rápidos. Segovia, Castro, Rico y Castro (1989) exponen algunos métodos y Gómez (1994) da también una relación amplia y detallada de estos métodos.

\section{Estimación y números sencillos o redondos}

La estimación produce resultados aproximados porque en los procesos de estimación se transforman o sustituyen los datos por números sencillos; es decir, que sean fáciles de memorizar y asequibles para las operaciones aritméticas mentales. Hay varias formas de producir, a partir de los datos exactos, números sencillos.

1) Números sencillos por truncamiento: truncar un número consiste en tomar sólo los dígitos de la izquierda más significativos según las situaciones. Para operar con el número resultante, se puede reemplazar por ceros las cifras suprimidas cuando son valores enteros (ejemplo, un truncamiento de 3572 sería 3570, ó 3500, ó 3000); o se puede operar con el número tal como queda y añadir posteriormente los ceros al resultado.

2) Números sencillos por redondeo: redondear un número, igual que el truncamiento, consiste en tomar sólo los dígitos de la izquierda más significativos de acuerdo a las diferentes situaciones, con la condición de que si la primera cifra que se desecha es $0,1,2,3$ ó 4, entonces la última cifra (y todas las demás) se mantienen igual; en otro caso, la última cifra que se mantiene aumenta en una unidad respecto del número que redondeamos; en el ejemplo anterior los posibles redondeos de 3572 serían 3570, 3600 y 4000. 
3) Números sencillos por sustitución: cuando un dato resulta complicado para operar con él, puede ser sustituido por otro próximo, con lo que desaparece la dificultad. Por ejemplo, para obtener un resultado estimado de $36894: 7$ se sustituye 36894 por 35000 de forma que la división 35000:7 resulta sencilla de realizar mentalmente.

\section{Estimación y aproximación}

La aproximación es un término de uso frecuente en cálculo numérico, que tiene relación con la estimación pero no es un sinónimo (Hall, 1984). Sowder (1989) también analiza la diferencia entre ambos términos. Más detalladamente, en Segovia, Castro, Rico y Castro (1989) se define la aproximación y su relación con la estimación. Aproximar es encontrar un resultado suficientemente preciso para un determinado propósito. La aproximación enfatiza la cercanía al valor exacto y es totalmente controlable; se aproxima tanto como la situación lo precise; tiene como herramientas los teoremas del cálculo (aproximado) o teoría de errores y los algoritmos de lápiz y papel o con calculadora. La estimación tiene en cuenta el error pero de manera menos precisa. A veces, éste no tiene un control asegurado. Las características de la estimación 3 y 6 citadas, no las tiene la aproximación. La estimación puede emplear algunos de los teoremas del cálculo aproximado en la medida que estos teoremas puedan aplicarse mentalmente. Hemos de decir que, en general, este trabajo no se refiere a la aproximación en los términos que acabamos de definir. Los términos aproximado y aproximación son usados con mucha frecuencia en nuestro trabajo para referirnos a los resultados de la estimación, que pueden calificarse de aproximaciones al valor exacto y por tanto aproximados, como también lo son los resultados de una aproximación en los términos que la hemos descrito; también nos referiremos a la aproximación como el proceso que consiste en sustituir un número por otro más sencillo y próximo.

\section{La estimación es parte de las matemáticas}

Una concepción simplista de las matemáticas asocia a éstas con la exactitud; y, por tanto, la estimación puede parecer ajena a las mismas o, más bien, una forma poco correcta de hacer matemáticas. El análisis de las razones que pueden provocar el uso de la estimación demuestra justamente lo contrario. "El uso de la estimación contiene los ideales matemáticos a saber: claridad de pensamiento y discurso, facilidad en relación con los problemas y consistencia en la aplicación de procedimientos" (Usiskin, 1986, p.2). 
Las razones por las que la estimación se hace necesaria pueden clasificarse en cinco grupos:

1) Imposibilidad de conocimiento de un valor exacto; como es el caso del empleo en algún cálculo de un valor desconocido de manera exacta; por ejemplo, el número de automóviles que salen de viaje un fin de semana.

2) Imposibilidad de tratamiento numérico exacto; por ejemplo, cuando empleamos en un cálculo un número decimal periódico.

3) Claridad numérica; los medios de comunicación, por ejemplo, para mayor claridad y comprensión de la información emplean estimaciones en lugar de cantidades exactas como "150 millones para una población escolar de 63 mil alumnos en lugar de "148739426 pesetas para una población escolar de 62879 alumnos".

4) Facilidad en el cálculo; son numerosas las situaciones en que la exactitud no es necesaria $\mathrm{y}$ es suficiente y útil un resultado aproximado; un redondeo apropiado y unos algoritmos mentales apropiados, proporcionan de manera sencilla un resultado bastante aproximado al exacto y útil para tomar decisiones.

\section{La estimación en el currículo escolar}

\section{Razones para la incorporación de la estimación al currículo escolar}

Hay dos razones fundamentales para que la Estimación se incorpore al currículo escolar. La primera es su utilidad práctica y la segunda es que completa la formación de los estudiantes. En relación a la primera razón, en la sección anterior ya se han dado algunos argumentos por los que la Estimación se hace necesaria en determinadas situaciones. También el conocido Informe Cockcroft (1985) destaca como complemento necesario a las necesidades matemáticas de los adultos: "La estimación puede considerarse desde distintas perspectivas. Un primer tipo de estimación es el que permite obtener, antes de efectuar un cálculo, una respuesta aproximada... que permita verificar si el resultado de una operación es del orden de magnitud correcto. Un segundo aspecto de la estimación es el que podría definirse como capacidad para determinar si la respuesta es o no razonable. Un aspecto conexo es la posibilidad de estimar medidas de diversos tipos, en el que sin duda la experiencia práctica y el uso continuado reportan los mejores resultados"(p.95). 
También el National Council of Teachers of Mathematics americano en su informe "An agenda for Action: Recomendations for School Mathematics of the 1980s", dice que la Estimación es útil en las tareas escolares cuando se usa como herramienta de comprobación de resultados o como recurso para la enseñanza de algunos tópicos; por ejemplo, la medida. También en los Estándares curriculares y de evaluación para la educación matemática del N.C.T.M. (1991) se considera que "las destrezas y estructuras conceptuales de la estimación potencian la capacidad que los niños tienen para enfrentarse a situaciones cuantitativas de la vida diaria" (p.35)

En relación a la segunda razón, la Estimación amplía la visión restrictiva de las matemáticas a la que antes hemos hecho referencia. La enseñanza escolar debe abarcar este doble carácter de la matemática, exacto y aproximado, y debe proporcionar a los estudiantes actividades que les permitan apreciar en qué circunstancias conviene utilizar una $\mathrm{u}$ otra. La estimación en definitiva "presenta a los estudiantes otra dimensión de las matemáticas; algunos términos como aproximadamente, casi, más cerca de, entre, un poco menos que, ilustran la idea de que las matemáticas implican algo más que exactitud" (N.C.T.M., 1991, p.36). La razón que resume todo tipo de argumentaciones en defensa de la enseñanza de la estimación la dan Edwards (1984, p.61) y Hope (1989, p.15): la Estimación desarrolla el Sentido Numérico.

La relación de componentes implicadas en la Estimación proporciona, por otro lado, una idea clara de la importancia de este tópico dentro del currículo escolar.

\section{Componentes implicadas en el cálculo estimativo:}

Sowder (1989, p.376) proporciona el siguiente listado:

\section{1) Componentes conceptuales:}

1.- Papel de los números aproximados.

1.1. Reconocer que la aproximación numérica se usa para calcular.

1.2. Reconocer que la estimación es un procedimiento con el que se obtienen valores aproximados.

2.- Multiplicidad de procesos/multiplicidad de resultados.

2.1 Aceptar más de un proceso para obtener una estimación.

2.2 Aceptar más de un valor como resultado de una estimación. 
3.- Conveniencias

3.1 Reconocer la conveniencia de que los procesos dependen del contexto.

3.2 Reconocer la conveniencia de estimar dependiendo del deseo de aproximar.

2) Componentes técnicas:

1. Procesos.

1.1 Reformulación: Cambiar los números usados para el cálculo.

- Redondeo

- Truncamiento

- Mediación

- Cambiar la expresión del número

1.2 Compensación: hacer ajustes durante o después del cálculo.

1.3 Traducción: cambiar la estructura del problema.

2. Resultados

2.1 Determinar en una estimación el orden de magnitud correcto.

2.2 Determinar una estimación aceptable.

3) Relacionar conceptos y técnicas:

1.- Habilidad para trabajar con potencias de 10.

2.- Conocer el valor posicional de los números.

3.- Habilidad para comparar números por tamaños.

4.- Habilidad para calcular mentalmente.

5.- Conocer factores básicos.

6.- Conocer las propiedades de las operaciones y su uso apropiado.

7.- Reconocer que modificar los números puede cambiar el resultado.

4) Componentes afectivas:

1.- Confianza en la habilidad para hacer matemáticas

2.- Confianza en la habilidad para estimar

3.- Tolerancia al error

4.- Reconocer que la estimación es útil. 
La estimación en el cálculo y en la medida: fundamentación curricular e investigaciones desarrolladas en el Departamento de Didáctica de la Matemática de la Universidad de Granada

\section{Incorporación de la estimación al currículo}

En Estados Unidos el interés por el tema de estimación no es reciente; los anuarios (yearbooks) del N.C.T.M. de los años 1937, 1960, 1976 y 1978 publican artículos sobre el tema; el de 1986 está íntegramente dedicado a estimación. El número de artículos publicados en revistas especializadas en matemática educativa en los 30 últimos años es muy elevado. A pesar del énfasis puesto en la incorporación del tema de estimación al currículo y de las investigaciones emprendidas, la situación en la enseñanza es presentada, de manera general por los investigadores, como de un bajo nivel de consolidación. Aunque el tema lleva años incorporado al currículo, sin embargo el tratamiento que se le da es muy superficial (Reys, 1984) y limitado (Hope, 1986, Johnson, 1979, Trafton, 1986, Sowder y Wheeler 1989). Carlow (1986) muestra en un estudio de un programa donde se incluye la estimación, y desarrollado a partir de 1969, la insatisfacción por los resultados obtenidos. Las pruebas de evaluación realizadas por el National Assessment of Educational Progress (NAEP) (Carpenter, Coburn y Reys, 1976; Montgomery, 1990) así lo pusieron de manifiesto.

En España la estimación aparece por primera vez de manera explícita en los diseños curriculares para los planes de estudios de Educación Primaria (MEC, 1992) y Secundaria Obligatoria (Nieto y otros, 1989). Concretamente, en Primaria uno de los objetivos es desarrollar la capacidad de "elaborar y utilizar estrategias personales de estimación, cálculo mental y orientación y aplicarlas en la resolución de problemas sencillos". Este objetivo general se desglosa en los objetivos de desarrollo de las capacidades siguientes (MEC, 1992, pp. 28-35):

1) Estimación de cantidades y del orden del resultado de operaciones.

2) Predicción y comprobación del resultado de operaciones y problemas.

3) Estimación y comprobación del resultado de mediciones.

4) Utilización de diferentes estrategias de contar de manera exacta y aproximada.

5) Decisión sobre la conveniencia o no de hacer cálculos exactos o aproximados en determinadas situaciones, valorando el grado de error admisible.

6) Estimación del resultado de un cálculo escogiendo entre varias soluciones propuestas y valoración de si una determinada respuesta numérica es o no razonable.

7) Elaboración de estrategias personales de cálculo mental con números sencillos.

8) Confianza en las propias capacidades y gusto por la realización personal de cálculo mental.

9) Valoración de la importancia de las mediciones y estimaciones en la vida cotidiana. 
En el actual Real Decreto de Mínimos de Primaria (MEC 2006) la estimación aparece referida al cálculo y a la medida. En le bloque I referido a Números y Operaciones se indica que "a lo largo de la etapa, se pretende que el alumno calcule con fluidez y haga estimaciones razonables, tratando de lograr un equilibrio entre comprensión conceptual y competencia en cálculo" y en el Bloque II, de La medida: estimación y cálculo de magnitudes se explicita "Estimación de resultados de medidas (distancias, tamaños, pesos, capacidades...) en contextos familiares".

De igual manera, para el caso de Secundaria Obligatoria, en el Real Decreto 1631/2006 de 29 de Diciembre se hace referencia a la estimación en el cálculo y en la medida; en el caso del cálculo se hace referencia al 'desarrollo de la capacidad de estimación y cálculo mental que facilite un control sobre los resultados y posibles errores; en el caso de la medida incluye la 'resolución de problemas que impliquen la estimación y el cálculo de longitudes, superficies y volúmenes'.

\section{Las investigaciones sobre estimación y la aportación del grupo de Pensamiento Numérico del Departamento de Didáctica de la Matemática de la Universidad de Granada}

A continuación, se describe una clasificación de las investigaciones sobre estimación, en primer lugar en dos grandes categorías, estimación en cálculo y estimación en medida y dentro de éstas, otras subcategorías en las que se incluyen ejemplos de investigaciones; se incluyen los trabajos realizados en el grupo de investigación Pensamiento Numérico y que son descritos con posterioridad.

\section{El caso del cálculo}

En Segovia (1987) y Segovia y Castro (2005) se hace una revisión de las investigaciones relacionadas con la estimación en cálculo que constituyen la base de las investigaciones que se han realizado en el Departamento de Didáctica de la Matemática de la Universidad de Granada; éstas pueden clasificarse en cuatro grandes categorías dentro de las cuales incluimos una revisión de investigaciones. 
La estimación en el cálculo y en la medida: fundamentación curricular e investigaciones desarrolladas en el Departamento de Didáctica de la Matemática de la Universidad de Granada

\section{Relación de la estimación con otras habilidades}

Bestgen y otros (1980) recopilan información sobre la actitud de los estudiantes de Magisterio ante la estimación en cálculo. Levine (1982) relaciona la habilidad en cálculo estimativo con el número y tipo de estrategias empleadas por estudiantes de collage. Rubenstein (1985) relaciona las estrategias de cálculo estimativo identificadas por Reys(1980) como, uso de números "compatibles", redondeo, etc. con otras técnicas matemáticas como la multiplicación y división, suma y resta y propone un conjunto de actividades relacionadas con la vida diaria en las que son muy apropiadas las estrategias referidas. Sowder (1989) y Sowder y Wheeler (1989) relacionan la estimación con un listado de componentes a las cuales se ha hecho referencia anteriormente. Lynchard (1989) estudia la relación de la estimación con el sentido numérico en alumnos de sexto grado que han recibido instrucción en estimación.

Case y Sowder (1990) relacionan la estimación en cálculo con el cálculo mental y la aproximación. Kinkade (1991) examina el rendimiento de los alumnos de octavo grado en estimación y describe la relación de las actitudes hacia las matemáticas y la estimación con el rendimiento en estimación. Gliner (1991) analiza distintas variables que pueden influir en el rendimiento en estimación en cálculo. Mottram (1996) analiza la influencia del contexto en la habilidad de estimar y en la elección de las estrategias de estimación. Albertson (1996) compara el rendimiento en estimación de alumnos con problemas de aprendizaje con otros alumnos.

Hanson y Hogan (2000) estudian la habilidad en estimación en cálculo de los alumnos universitarios. De Castro, Castro y Segovia (2002) analizan la influencia del tipo de número en la resolución de tareas de estimación.

\section{Comparación de métodos de enseñanza}

Bestgen y otros (1980) comprueban que la instrucción en técnicas de estimación de soluciones de problemas en alumnos de los estudios de Magisterio mejora su capacidad para estimar y su actitud hacia la estimación. Jarret (1980) comprueba el efecto de tres métodos de enseñanza en los resultados de la estimación. Shoen y otros (1981) analizan los efectos de diferentes programas de enseñanza de la estimación en cálculo con números naturales en los grados $4^{\circ}, 5^{\circ}$ y $6^{\circ}$ poniéndose de manifiesto una mejora en el rendimiento en actividades de estimación a nivel de ejercicios y de problemas. Abed (1985) compara la efectividad de tres métodos de enseñanza de la estimación del cociente de divisiones con números decimales. 
Segovia (1986) también pone de manifiesto que la instrucción en cálculo estimativo mejora el rendimiento en éste, en relación a niños que no la reciben. Whalen (1989) compara dos métodos de instrucción de estimación en cálculo.

Sanfiorenzo (1990) realiza un estudio experimental en el que compara la efectividad de tres métodos de enseñanza de la estimación con números decimales. Chien (1990) intenta demostrar que las destrezas de estimación en cálculo de maestros en formación pueden mejorar si éstos utilizan materiales adecuados de auto-aprendizaje. Fernández-Cano (1991) compara dos métodos de instrucción, con y sin calculadora y analiza sus efectos sobre el cálculo mental y la estimación en niños de $3^{\circ}$ de EGB.

\section{Identificación de estrategias}

Reys y otros (1982) identifican las estrategias de cálculo estimativo que realizan los buenos estimadores de $7^{\circ}$ a $12^{\circ}$ grado y que clasifican en Procesos de Reformulación (Front-end, uso de números Compatibles, Redondeando y Truncando), Procesos de Traducción y Procesos de Compensación. Wyatt (1986) identifica los procesos utilizados en las estimaciones, la razonabilidad de las estimaciones y los criterios de razonabilidad. Brame (1986) investiga las estrategias empleadas por alumnos de secundaria que son considerados como malos estimadores. Sowder y Wheeler (1989) analizan las respuestas de los niños de los grados 3, 5, 7 y 9 en tareas de estimación desde el punto de vista de las estrategias y resultados y su relación con un extenso listado de componentes implicadas en el cálculo estimativo y a las que ya se ha hecho referencia.

Morgan (1990) muestra que el contexto puede propiciar el desarrollo de estrategias de estimación. Flores, Reys y Reys (1990) identifican estrategias en tareas de estimación de cálculo empleadas por niños mexicanos en el marco conceptual desarrollado por Reys; sus resultados proporcionan una validación del marco teórico general usado en las investigaciones de Reys (1982). Segovia, Castro, Rico y Castro (1989) elaboran un organigrama que incorpora todas las posibles estrategias descritas por Reys y que permite un análisis de la resolución de tareas de estimación. Shoen, Blume y Hoover (1990) analizan la realización por estudiantes de $5^{\circ}$ a $8^{\circ}$ de un test de estimación con diferentes formatos: elección múltiple, orden de magnitud, rango y punto de referencia entre otros. Dowker (1992) explora la precisión y estrategias empleadas en la estimación de una muestra de matemáticos profesionales. Reehm (1994) identifica los procesos de estimación de alumnos de octavo grado cuando estiman problemas en formato numérico y 
La estimación en el cálculo y en la medida: fundamentación curricular e investigaciones desarrolladas en el Departamento de Didáctica de la Matemática de la Universidad de Granada

contextual. Dowker (1992) comparan las estrategias de estimación de matemáticos profesionales con otros tres colectivos: contables, estudiantes de psicología y estudiantes de inglés.

\section{Instrucción y evaluación}

Levin (1981) propone una serie de técnicas de estimación basadas en el cálculo mental, el concepto de medida y el número real. Por ejemplo, una técnica de cálculo de $0,7 \times 0,5$ es representar mentalmente, sobre una cantidad que se toma como unidad, el 70\% y sobre éste el $50 \%$; de esta forma determinamos el resultado aproximado en relación a la cantidad inicial. Reys (1984, 1988) da orientaciones para la enseñanza y evaluación de la estimación. Para la instrucción, el uso de estrategias debe adaptarse a la evolución tradicional del currículo: números enteros, decimales, fracciones y porcentajes. La evaluación debe enfatizar la naturaleza mental de la estimación; el control del tiempo y el empleo de formatos diferentes para la elaboración de los tests son también aspectos importantes. Edwars (1983) desarrolla e implementa un programa para la enseñanza de la estimación en cálculo para adultos. Edwards (1984) analiza las causas del fracaso de la enseñanza de la estimación: multiplicidad de métodos de estimación, evaluación difícil y la consideración, por el alumnado e incluso el profesorado, que la estimación es una forma de hacer matemáticas "inferior". Reys, Bestgen, Trafton y Zawojewski (1984) desarrollan unas lecciones de estimación en cálculo, las implementan y evalúan los resultados. Rubenstein (1985) propone actividades que desarrollan las estrategias identificadas por Reys y otros (1980).

Segovia (1986) elabora un test de estimación en cálculo para evaluar a niños de $6^{\circ}$ curso a los que se han impartido unas lecciones de estimación en suma y resta: consta de 21 ítems de elección múltiple, 14 de suma y resta en los que se alterna la necesidad de "llevarse" en los cálculos exactos de las operaciones, ninguna vez, una vez o dos veces. Con los siete ítems de multiplicación y división se pretende observar si el niño es capaz de desarrollar técnicas propias a partir de las aprendidas en las otras operaciones. Gossard (1986) evalúa la enseñanza recibida sobre estimación comparando lo enseñado con lo aprendido. Markovits (1987) analiza la influencia de un tratamiento en estimación corto (una lección) en niños de sexto y séptimo grado; los estudiantes mejoran significativamente su rendimiento en estimación después de la instrucción recibida. Whiteman (1989) analiza la influencia de un periodo de instrucción sobre estimación en el aprendizaje de estrategias y en la habilidad de estimar. Sliva (1988) elabora un test de estimación con ítems de respuesta abierta para evaluar la habilidad de estimación en cálculo. 
Goodman (1991) desarrolla un test de estimación para evaluar alumnos de los estudios de magisterio y comparar los de menor rendimiento con los de mayor rendimiento. Bobis (1991) investiga el efecto de la enseñanza de la estimación en la habilidad de estimar y en el desarrollo de estrategias de estimación. Murphy (1992) estudia el efecto de la instrucción en la habilidad de estimar. Smith (1993) evalúa la comprensión de las estrategias de estimación en cálculos de suma y resta de estudiantes de magisterio. Floyd (1994) realiza un estudio experimental para evaluar el efecto de dos secuencias de instrucción sobre estimación en cálculo de fracciones en el rendimiento de los alumnos en estimación. Clayton (1996) realiza un análisis del papel del porcentaje de error en la evaluación de tareas estimación en diferentes investigaciones. Heinrich (1999) analiza la habilidad en el uso de estrategias de estimación antes y después de un periodo de instrucción.

\section{La estimación y el cálculo aproximado en la EGB}

Dentro de este último grupo de trabajos se encuentra La estimación y el cálculo aproximado en la EGB (Segovia, 1986). Este trabajo pone de manifiesto que "el desarrollo de unos contenidos orientados hacia los conceptos de aproximación-estimación con el empleo y práctica de unas técnicas de cálculo en un tiempo relativamente breve y que no entorpece la enseñanza de otros contenidos, mejora el rendimiento de los alumnos en problemas de cálculo donde se pide una respuesta aproximada obtenida de forma mental". Para ello se elaboran unas lecciones de estimación en cálculo basadas en el empleo del redondeo y en el uso de técnicas de cálculo mental que se desarrollan en el curso; estas lecciones son:

Lección 1: Número exacto y número aproximado. Redondeo de un número. Error en redondeo. Tipos de redondeo. Orden de aproximación de un redondeo. Comparación de errores.

Lección 2: Utilidad de la estimación. Técnicas de suma. Técnicas de resta.

Lección 3: Redondeo en sumas. Técnicas sumas aproximadas. Técnicas de restas aproximadas.

También se elaboró un cuestionario de estimación para medir el rendimiento de los alumnos comparados con otros alumnos que no habían recibido el tratamiento. El cuestionario utilizado puede verse en el Anexo I.

\section{Diagrama de estrategias de estimación en cálculo}

Dentro del tercer grupo de trabajos, en Segovia, Castro, Rico y Castro (1989, p. 151152) se resume mediante un diagrama los procesos que resultan de las investigaciones de $R$. 
E. Reys, Bestgen, Rybolt, \& Wyatt (1982); este diagrama (Anexo II) constituye un buen instrumento de análisis de las posibles estrategias de estimación que posteriormente ha sido empleado en otras investigaciones como en De Castro, Segovia y Castro (2002).

Influencia del tipo de número en la estimación en cálculo

De Castro, Castro y Segovia (2002), apoyándose en los resultados de Levine (1980) y Morgan (1990) plantean la hipótesis de que la verdadera diferencia de dificultad en las tareas de estimación se da entre los ítems que tienen números enteros o decimales mayores que uno y aquellos en los que aparecen números decimales menores que uno. En su investigación participan 53 maestros en formación a los que se les administra el test de Levine (1982) (Anexo 3), tras un periodo de instrucción sobre estimación de 10 horas (de Castro, 2001, p.269-315). Se llega a la conclusión de que estimar con decimales menores que uno es más difícil que con enteros o decimales mayores que uno, lo que confirma la hipótesis planteada.

En el trabajo se exploran además la influencia del tipo de operación en el resultado y las estrategias de estimación que emplean los sujetos aplicando el modelo referido en la investigación del apartado anterior; los resultados de este análisis muestran que los sujetos usan una variedad de estrategias que van desde aquellas que emplean uno sólo de los procesos hasta estrategias de estimación que incorporan todos los procesos.

\section{El caso de la medida}

En Joram, Subrahmanyam y Gelman (1988) se distinguen tres generaciones de investigaciones sobre estimación en medida relacionadas con las tareas de estimación. En la primera generación los investigadores se interesaron solamente por las respuestas numéricas que daban los sujetos en las tareas de estimación. Por ejemplo, en una de las investigaciones de este tipo, se pidió a los individuos que estimaran las medidas de objetos familiares no presentes en el momento de la estimación como podía ser la altura de una puerta; las respuestas se catalogaban como 'razonables', 'incorrectas' y 'correctas'. Se observó que los resultados mejoraban con la edad.

En la segunda generación las investigaciones sen centraron en las diferencias entre los sujetos en la habilidad para estimar atributos como el peso o la altura estando la cantidad a estimar presente o ausente; la catalogación de las respuestas introdujo el porcentaje de error aunque en algunas investigaciones se siguió utilizando la clasificación de respuesta 'correcta', 
'razonable' o 'incorrecta'. Se observaron mejoras en la precisión con la edad; las estimaciones de medidas longitudinales fueron más precisas que las de peso, capacidad o volumen.

La tercera generación pone de manifiesto el interés por los procesos cognitivos implicados en las estimaciones así como en las diferentes estrategias empleadas.

En Segovia (1996) se hace una clasificación atendiendo al tipo de cantidad que se estima, continua o discreta; en cada caso se presenta una revisión de la literatura de investigación.

\section{Magnitudes continuas}

Corle (1963) analiza las estimaciones sobre cantidades de longitud, tiempo, peso, etc. que realizan estudiantes de magisterio. Hildreth (1983) identifica estrategias empleadas por alumnos de $5^{\circ}, 7^{\circ}$ y college en tareas de estimación de longitud y área. Siegel, Goldsmith y Madson (1985) analizan las habilidades de los niños en estimación de cantidades y proponen un modelo que recoge las diferentes estrategias de estimación en medida. Bright (1979) analiza la influencia de la práctica en la estimación de cantidades lineales poniendo de manifiesto que ésta es significativa; no es significativa, sin embargo, la influencia de la estimación física en la estimación simbólica (empleo de unidades de medida no convencionales). Markovits (1987) analiza las respuestas de los estudiantes en tareas de estimación. Clayton (1988) muestra que las estimaciones tienen un error de un $20 \%$ cuando las cantidades son inferiores a 100.

Forreste (1990) estudia el papel del contexto en la estimación de los niños de Primaria (de 5 a 8 años) en tareas de estimación sobre distancia, área y volumen. Callis (2002) realiza una investigación que aporta conocimiento sobre la capacidad estimativa métrica. Sus objetivos se centran tanto en la detectar los procedimientos como los recursos y estrategias que se aplican, analizándose a la vez, su incidencia de uso y su eficacia o nivel de precisión; y también cómo se organizan y generan estas estructuras mentales estimativas. Castillo (2006) elabora un cuestionario de estimación de cantidades de longitud, superficie, capacidad y peso y detecta grandes errores de estimación; describe también las estrategias que emplean los sujetos en sus estimaciones.

\section{Magnitudes discretas}

Distinguimos en este caso entre las estimaciones que exigen una respuesta numérica y aquellas de comparación (numerosidad relativa): 
1) Con respuesta numérica: Siegel, Goldsmith y Madson (1985) proponen un modelo de elaboración de estrategias de estimación de cantidades, entre las que se incluye la numerosidad. Crites (1989) identifica las estrategias de estimación, así como la rentabilidad de las mismas, empleadas por los niños de $3^{\circ}, 5^{\circ}$ y $6^{\circ}$ grado. Barody y Gatzke (1991) estudian la relación de las estimaciones en niños de preescolar con los valores de la cantidad: para conjuntos de más de ocho elementos las estimaciones tienen un error superior al $25 \%$; la instrucción en estimación puede ser rentable cuando se utilizan cantidades de elementos inferiores a diez. Lentzinger, Rathmell y Urbatsch (1986) muestran que la percepción visual en niños de 6 años es una limitación para la estimación: en esta edad la cantidad de treinta y la cantidad de cinco y mil son similares; las dos son muy grandes. Para mejorar el rendimiento en estimación sugiere que se favorezca el empleo de estrategias de comparación con cantidades conocidas, partición en cantidades conocidas y el uso del cálculo mental. Krueger (1972) relaciona la respuesta dada sobre estimación y la cantidad real (estímulo); los tiempos de visualización son muy cortos; se analiza exclusivamente la percepción de la cantidad en instantes de segundo.

Karkovits y Hershkowitz (1993) identifican las estrategias de estimación empleadas por niños de tercer grado y la influencia en estas estrategias de la realización de actividades de estimación. Segovia (1997) identifica las estrategias de estimación empleadas por estudiantes de primer a octavo curso (6 a 14 años) y analiza la evolución con la edad de la resolución de las tareas de estimación. Pareja (2002) aplica el mismo cuestionario que Segovia (1997) con estudiantes de Magisterio para identificar estrategias de ejecución y analizar los errores.

2) Numerosidad relativa: Piaget y Szeminska (1964) ponen de manifiesto que la longitud influye en los juicios sobre numerosidad que realizan los niños. McLaughin (1981) muestra que los niños de menos de 7 años implican la longitud y la densidad en sus juicios de comparación. Según Cuneo (1982) los niños en una primera etapa integran la longitud y la densidad de manera aditiva en tareas de estimación de numerosidad y más tarde lo hacen de una manera multiplicativa. Para Cowan (1987) las estimaciones fundamentadas en el conteo por los niños de 6 años son inconsistentes. Howe y Jund (1987) nos enseñan que la simetría de la configuración de la cantidad influye en el resultado de las estimaciones. 


\section{Estimación de cantidades discretas}

En Segovia (1997) la investigación explora y analiza los procesos mentales que los niños de 6 a 14 años emplean cuando resuelven tareas de estimación de cantidades discretas, así como los resultados y la evolución de procesos y resultados con la edad. Las tareas consistieron en estimar la cantidad de objetos (circulitos) que aparecían en la pantalla de un ordenador durante un tiempo limitado que no permitiese a los sujetos la opción de contar; los objetos se disponían según determinadas estructuras geométricas y tenían distintos tamaños; las respuestas se escribían en el teclado del ordenador y éste almacenaba la respuesta y el tiempo en emitirla; al mismo tiempo se preguntaba al sujeto el procedimiento que había empleado para obtener el valor estimado en 4 de las 16 tareas de las que constaba la prueba.

Se obtuvo que la precisión en los resultados evoluciona con la edad que va desde un error superior al $50 \%$ en primer curso, en torno a un $30 \%$ en segundo y tercero, en torno a un $20 \%$ en cuarto y quinto y sexto y en torno a un $15 \%$ en séptimo y octavo; la precisión de los resultados depende del tamaño de la cantidad; a mayor cantidad menor precisión; la estructura de la cantidad influye en la precisión de la estimación; se identificaron 12 estrategias de estimación agrupadas en diferentes categorías; estas estrategias también estaban asociadas a distintos niveles de edad; a definición de cada una de las doce estrategias, junto con la codificación que le asignamos, se presenta a continuación. Estas estrategias las presentamos agrupadas en cuatro categorías generales.

\section{1) No justificadas.}

Estrategia 1: No justificada. En este tipo incluimos aquellos procedimientos en los que el niño no es capaz de explicitar ningún argumento o dice por ejemplo "no lo sé".

2) Valoración global sin referente.Estas estrategias se caracterizan porque el niño considera la cantidad presentada globalmente, es decir, no considera una parte de la cantidad propuesta para hacer una valoración del total.Teniendo en cuenta que el niño realice la valoración empleando la secuencia numérica o no, contando sobre la imagen real o mental, y tenga en cuenta el tamaño como criterio de valoración o no, tendremos las diferentes estrategias.

Estrategia 2: Recitar la secuencia numérica sin considerar la cantidad. El procedimiento consiste en enunciar la secuencia numérica sin asociar los números a los elementos de la cantidad, parándose en un número sin criterio relacionado con la cantidad. 
Estrategia 3: Recitar la secuencia numérica según el tamaño. Consiste en enunciar la secuencia numérica parándose en un número que él relaciona con el tamaño numérico o espacial.

Estrategia 4: Asignar un número sin considerar la cantidad. El niño explicita un criterio que no implica una acción de cardinación razonada: "me lo he inventado", "se me ha venido a la cabeza", "lo he pensado", etc.

Estrategia 5: Asignar un número según el tamaño. El niño asigna un número grande o pequeño según el tamaño numérico o espacial de la cantidad: "porque era muy grande", "porque es pequeña", "porque hay muchos", etc.

Estrategia 6: Contar la cantidad real o mental. El niño cuenta la cantidad de circulitos mientras permanece la imagen de la figura en la pantalla del ordenador y si no tiene tiempo de contarlos íntegramente durante ese tiempo, sigue contando sobre una imagen mental.

3) Estrategias que implican una valoración global de la cantidad mediante comparación con un referente

Estrategia 7: Asignar un número por comparación. En este caso el niño asigna un número a la cantidad por comparación con otra que ha visto en alguna de las tareas propuestas previamente.

4) Estrategias que implican una valoración parcial de la cantidad. Estas estrategias se pueden clasificar en dos subgrupos:

a) Sin descomposición previa de la cantidad:

Estrategia 8: Contar una parte y estimar según el tamaño.El niño cuenta mientras permanece la imagen en pantalla y deduce el total sin dar una justificación o con criterios simples como "porque es muy grande", "porque es pequeño", etc.

Estrategia 9: Contar una parte, estimar el resto y sumar. El niño cuenta mientras la imagen de la cantidad permanece en pantalla, estima el resto por comparación con la parte contada y suma. Por ejemplo, el niño dice "he dicho 23 pues he contado 13 y 10 que creo que hay también". 
Estrategia 10: Iterar una parte sobre el total. El niño cuenta, subitiza o estima una cantidad de elementos, por ejemplo 5, itera la longitud de los mismos sobre el total y mediante sumas parciales, $(. .(((5+5)+5)+5)+\ldots)+5$, obtiene el resultado.

b) Con descomposición previa de la cantidad

En este caso los procesos son más complejos, y en general, constan de tres componentes: definición de una parte y su relación con el total, determinación del número de elementos de esa parte y recomposición del total.

Estrategia 11: Determinar la mitad y duplicar. El niño cuenta hasta la mitad o intenta contarla mientras permanece la imagen en la pantalla, determina la parte restante mediante comparaciones de tipo "igual más o menos", "unos pocos más", etc y obtiene el total sumando o multiplicando por dos.

Estrategia 12: Contar una parte y multiplicar o sumar . El niño descompone la cantidad en tres o más partes iguales y reconstruye el total sumando o mediante multiplicación del número obtenido en la determinación de la parte por el número de partes.

\section{Estimación de cantidades discretas por alumnos de magisterio}

En Pareja (2001) se realiza una réplica de la investigación anterior con estudiantes de Magisterio obteniéndose una mejora en la precisión de las estimaciones así como en las estrategias utilizadas aunque no se aportan nuevas estrategias a las descritas en la investigación anterior.

\section{Estimación de cantidades continuas: longitud, superficie, capacidad y masa}

En Castillo (2006) se plantean los siguientes objetivos de investigación relativos a la estimación por estudiantes de Magisterio y Enseñanza Secundaria:

a) Describir y caracterizar el error de las estimaciones de cantidades de magnitudes continuas, Longitud, Superficie, Capacidad y Masa/peso realizadas por los sujetos implicados en el estudio.

b) Describir y caracterizar los diferentes procesos de estimación empleadas por los sujetos. 
Con este fin se elaboró un cuestionario en el cuál se les pedía a los alumnos que realizaran diversas estimaciones y que escribieran cual había sido el procedimiento y/o razonamiento que habían seguido para obtenerlas. Dicho cuestionario constaba de cuatro preguntas:

1. Estimar la longitud de la mesa del profesor (en $\mathrm{cm}$ ).

2. Estimar la superficie de la pizarra $\left(\mathrm{en}^{2}\right)$.

3. Estimar la capacidad de la papelera (en litros).

4. Estimar el peso de una silla (en Kg.).

La tabla 1 recoge las medias de las estimaciones, así como los porcentajes medios de error que los alumnos de cada grupo cometieron para cada uno de las estimaciones.

Tabla 1. Medias de las estimaciones y porcentajes medios de error

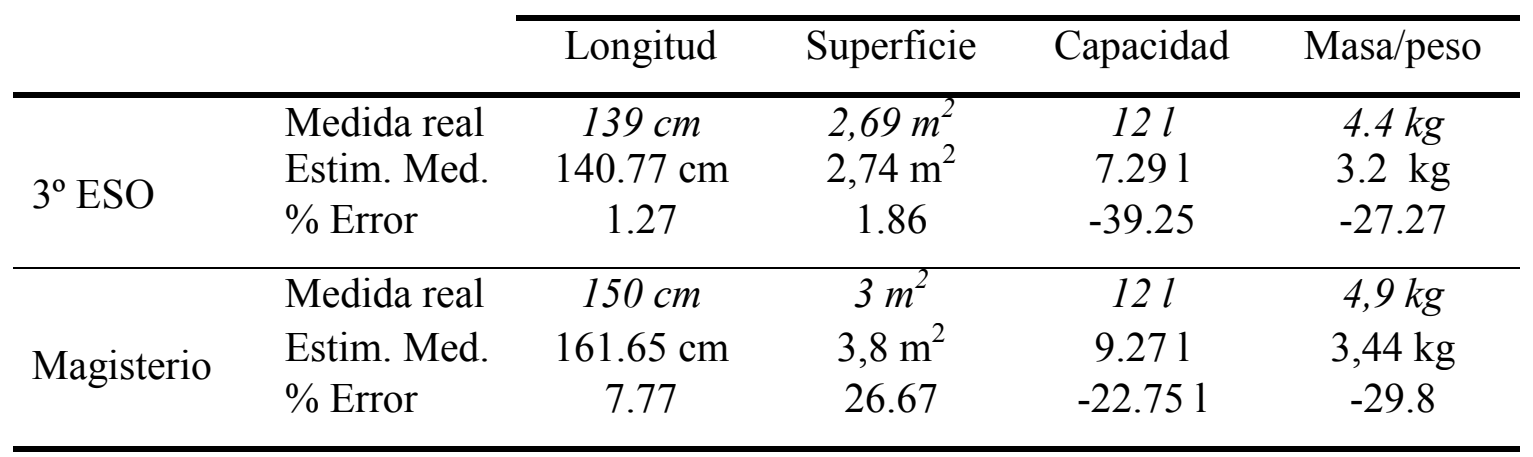

Para el caso de las magnitudes Longitud y Superficie, para los dos grupos de alumnos, se presenta una tendencia a la sobreestimación (es decir, estimar por encima del valor real). En el caso de la Capacidad y la Masa, por el contrario, la tendencia es a la subestimación (estimar por debajo del valor real). Para realizar la identificación y clasificación de procesos se consideró la idea de diferenciar entre si el referente (cantidad de un objeto conocido que se emplea para comparar con la cantidad que se pretende estimar) estaba presente o ausente, Bright (1976).

Los procesos empleados fueron: a) No contesta (el alumno no da respuesta); b) 'A ojo' (el alumno explica que lo ha hecho 'a ojo'); c) Iterando un referente presente;d) Iterando un referente ausente; e) Acotando (el valor se ubica entre dos cantidades); f) Comparando la cantidad a estimar con un referente aproximadamente igual (referente presente); g) Comparando la cantidad a estimar con un referente aproximadamente igual. (referente 
ausente); h) Comparando la cantidad a estimar con un múltiplo de un referente (referente presente); i) Comparando la cantidad a estimar con un múltiplo de un referente (referente ausente); j) Comparando la cantidad a estimar con un divisor o fracción de un referente (referente presente); k) Comparando la cantidad a estimar con un divisor o fracción de un referente (referente ausente); 1) Descomponiendo/Recomponiendo en partes iguales (se descompone la cantidad, luego se estima cada parte y se obtiene el total); m) Descomponiendo/Recomponiendo en una parte más su complementario; $\mathrm{n}$ ) Descomponiendo/Recomponiendo en partes diferentes; o) Técnica indirectas (empleo de fórmulas); q) Reajuste (se estima y luego se realizan reajustes del valor obtenido).

\section{Conclusiones}

Hemos presentado una revisión de la estimación desde el punto de vista curricular y de investigación, destacando las aportaciones que se han realizado dentro de nuestro grupo de trabajo; en el caso de la investigación lo hemos hecho en distintas perspectivas: estrategias de estimación en cálculo, efectos de la instrucción en la estimación en cálculo, rendimiento y estrategias de estimación en medida de cantidades discretas en diferentes niveles, rendimiento y rendimiento y estrategias de estimación en medida de cantidades continuas. En estas y otras investigaciones así como en nuestra experiencia como docentes, se pone de manifiesto la dificultad de incorporar la estimación al currículo de la enseñanza obligatoria; éste es un campo abierto a la investigación con cuestiones por resolver como:

¿Cuál es el procedimiento más adecuado de incorporar la estimación al currículo? ¿En qué niveles de la enseñanza obligatoria es más apropiado el trabajo de estimación y cómo? ¿Cuál es la forma más adecuada de evaluar las producciones de los alumnos en estimación?

Con la idea de dar respuesta a algunas de estas interrogantes, nuestro grupo de trabajo, apoyándonos en los resultados de Castillo (2006) continúa trabajando en este tópico. Estamos aplicando y comprobando los efectos de un plan de actuación en el aula de secundaria, diferenciado del que se suele recoger en los libros de texto, sobre estimación de magnitudes continuas, en el caso concreto de la longitud y la superficie. 
La estimación en el cálculo y en la medida: fundamentación curricular e investigaciones desarrolladas en el Departamento de Didáctica de la Matemática de la Universidad de Granada

\section{Referencias}

Abed, A. S: (1985). An evaluation of the suitability of two decimal division quotient estimation techniques for seventh graders, and their effect upo calculation errors. (Doctoral dissertation, The Florida Satet University, (1985). Dissertation Abstracts Intenational, 46A, 1548. (DA8517333).

Albertson, A. M. (1996). Computational estimation: A comparisom of learning disabled students and controls. (Master's thesis, MGH Institute of Health Professions, 1995). Masters Abstracts International, 34/04, 1353. (DA1378541)

Barody, A. y Gatzke, M. (1991). The estimation of set size by potentialially giften kindergartenage children. Journal for Research in Mathematics Education, 22 (1), 59-68.

Benton, S. (1986). A summary of research on teaching and learning estimation. In H. L. Schoen, \& M. J. Zweng (Eds.), Estimation and mental computation (pp. 16-30). Reston, VA: NCTM.

Bestgen, B., Reys, R., Rybolt, J., \& Wyatt, J. W. (1980). Effectiveness of systematic instruction on attitudes and computational estimation skills of preservice elementary teachers. Journal for Research in Mathematics Education. 11(2), 124-136.

Bobis, J. (1991). The effect of instruction on the development of computational estimation strategies. Mathematics Education Research Journal, 3(1), 17-29.

Brame, O. H. (1986). Computational estimation strategies used by high school students of limited computational estimation ability. (Doctoral dissertation, North Texas State University, 1986) Dissertation Abstracts International, 47A, 1228. (DA8616027)

Bright, G.W. (1979). Measuring experienced teachers' linear estimation skills at two levels of abstraction. School Science and Mathematics 79, 161-64.

Carlow, C. (1986). Critical balances and payoffs of an estimation program. En H.L. Schoen \& M.J. Zweng (Eds.). Estimation and mental computation (pp. 93-102). NCTM. Reston, VA.

Carpenter, T. P., Coburn, T. G., Reys, R. E., \& Wilson, J. W. (1976). Notes from national assessment: Estimation. Arithmetic Teacher, 23(4), 296-302.

Case, R. (1985). Intellectual development. Orlando, Fla.: Academic Press.

Case, R., \& Sowder, J. (1990). The development of computational estimation: A neopiagetian analysis. Cognition and Instruction, 7(2), 79-104.

Castillo, J. J. (2006). Estimación de cantidades continuas: longitud, superficie, capacidad y masa (Memoria de Tercer Ciclo). Departamento de Didáctica de la Matemática de la Universidad de Granada. 
Callís, J. (2002). Estimació de mesures longitudinals rectilínies i curvilínies. Procediments, recursos I estratégies. Tesis Doctoral. Departamento de Didáctica de la Matemática y de las Ciencias Experimentales. Universidad Autónoma de Barcelona.

Clayton, J.G. (1988). Estimation. Mathematics Teaching, MT125 (Dic), 18-19

Clayton, J. G. (1996). A crtiterion for estimation task. International Journal of Mathematical Education in Science and Technology, 27(1), 87-102.

Chandler, D. G., \& Brosnan, P. (1994). Mathematics textbook changes from before to after 1989. Focus on Learning Problems in Mathematics, 16(4), 1-9.

Chien, Y. C. (1990). The use of self-study material for improving the computational estimation skills of preservice teachers. (Doctoral dissertation, Columbia University Teachers College, 1990) Dissertation Abstracts International, 51A, 774. (DA9021279).

Cockcroft, W.H. (1985). Las matemáticas sí cuentan. Informe Cockcroft. MInisterio de Educación y Ciencia. Madrid.

Corle, C.G. (1963). Estimates of quantity by elementary teachers and college juniors. Arithmetic Teacher, 10(2), 347-353.

Cowan, R. (1987). When do Children trust counting as a basis for relative number judgments?. Experimental child psichology, 43, 328-345.

Crites, T. W. (1989). Identification and characterzation of the strategies used by third-, fifth-, and seventh-grade students when making estimates of numerosity. Ph. D. University of Missouri - Columbia. Supervisor: Reys R. Order Number DA9010543.

Cuneo, D. O. (1982). Children's judgments of numerical quantity: A new view of early quantification. Cognitive psychology, 14, 13-44.

De Castro, C. (2002). Influencia del tipo de número en la estimación en cálculo. Granada: Departamento de Didáctica de la Matemática. Universidad de Granada.

De Castro, C., Castro, E., \& Segovia, I. (2002). Influence of number type and analysis of errors in computational estimation tasks. In A. D. Cockburn, \& E. Nardi (Eds.), Proceedings of the $26^{\text {th }}$ Conference of the International group for the Psychology of Mathematics Education (vol. 2, pp. 201-208). Norwich, UK.

De Castro, C., Segovia, I., \& Castro, E. (2002). An alternative model for the description of computational estimation strategies. In A. D. Cockburn, \& E. Nardi (Eds.), Proceedings of the $26^{\text {th }}$ Conference of the International group for the Psychology of Mathematics Education (vol. 2, pp. 193-200). Norwich, UK.

Dowker, A. (1992). Computational estimation strategies of professional mathematicians. Journal for Research in Mathematics Education, 23(1), 45-55. 
Dowker, A. (1997). Young children's addition estimates. Mathematical Cognition, 3(2), 141154.

Dowker, A., Flood, A., Griffiths, H., Harris, L., \& Hook, L. (1996). Estimation strategies of four groups. Mathematical Cognition, 2(2), 113-135.

Edwards. A. (1983). Estimation for numeracy. Numeracy project. Boroko: Administrative College of Papua New Guinea.

Edwards, A. (1984). Computational estimation for numeracy. Educational Studies in Mathematics, 15(1), 59-73.

Fernádez-Cano, A. (1991). Impacto de la calculadora electrónica en la educación matemática.

Un estudio cuasi-experimental en tercer nivel. Tesis Doctoral. Universidad de Granada. Departamento de Pedagogía.

Flores, A., Reys, B. J. and Reys, R. E. (1990). Desempeño y estrategias en la estimación en operaciones aritméticas de alumnos de quinto de primaria y segundo de secundaria en México. Educación Matemática, 2 (1), 30-44.

Floyd, T. (1994). A compariso of two instructional sequences for the teaching of estimation of fraccional computation to fifth-grade students. (Doctoral dissertation University of Missouri-Columbia, 1992). Dissertation Abstracts International, 54/07, $2498 \mathrm{~A}$. (DA9400024).

Gliner, G. S. (1991). Factors contributing to success in mathematical estimation in preservice teachers: Types of problems and previous mathematical experience. Educational Studies in Mathematics, 22(6), 595-606.

Greeno, J.G. (1991). Number sense as situated knowing in a conceptual domain. Journal for Research in Mathematics Education, 22(13), 170-218.

Gómez, B. (1994). Los métodos de cálculo mental en el contexto educativo y los procesos cognitivos involucrados en los errores que cometen los estudiantes al aplicarlos. Tesis doctoral. Departamento de Didáctica de la Matemática. Universidad de Valencia.

Goodman, T. (1991). Computational estimation skills of pre-service elementary teachers. International Journal of Mathematical Education in Science and Technology, 22, 259272.

Hall, L.(1984). Estimation and aproximation not synonyms. Mathematics Teacher. Oct. 1984. 516-517.

Hanson, S. A., \& Hogan, T. P. (2000). Computational estimation skill of college students. Journal for Research in Mathematics Education, 31(4), 483-499. 
Heinrich, E. J. (1999). Characteristics and skills eshibited by middle school students in performing the task of computational estimation. (Doctoral dissertation, Fordham University, 1998). Dissertation Abstracts Intenational, 59/07, 2405A. (DA9839507).

Hildreth D.J. (1983). The use of strategies in estimating measurements. Arithmetic Teacher, 30(5), 50-54.

Hope, J. (1989). Promoting number sense in school. Arithmetic Teacher, 36(6), 12-18.

Howden, H. (1989). Teaching number sense. Arithmetic Teacher, 36(6), 6-11.

Howe E. y Jung K. (1987). Judgments of Numerosity: Effects of Symmetry and Goodness in dot Pattern Arrays. Acta Psychologica 64, 3-11.

Jarrett, J. A. (1980). A study of the differential effects of three levels of instruction in estimation on fifteh and sixth grade pupils. (Doctoral dissertation, University of Iowa, 1980). Dissertation Abstracts International, 41/04, 1452A. (DA8022038).

Johnson, D. (1979). Teaching estimation and reasonableness of results. Aritmetic Teacher. September 1979, pp.34-37.

Joram, E., Subrahmanyam, K. and Gelman, R. (1998): Measurement estimation: Learning to map the route from number to quantity and back. Review of Educational Research, 68(4), 413-449.

Kimkade, S. S. (1991). An analysis of the performance of United Sates eigth-grade classes on estimation and approximation items from the Secondo International Mathematics Study (SIMS) - a secondary analysis. (Doctoral dissertation, Southern Illinois University at Carbondale, 1990). Dissertation Abstracts International, 52/05A, 1673. (DA9129843).

Koyama, M. (1994). Research into relationship between the computational estimation ability and strategy and the mental computation ability: Analysis of a survey of the fourth, fifth, and sixth graders in Japan. Hiroshima Journal of Mathematics Education, 2, 3544.

Krueger L.(1972). Perceived numerosity. Perception and Psychophysics, 11 (1A).

LeFevre, J., Greenham, S. L., \& Waheed, N. (1993). The development of procedural and conceptual knowledge in computational estimation. Cognition and Instruction, 11(2), 95-132.

Lemaire, P., Lecacheur, M., \& Farioli, F. (2000). Children's strategy use in computational estimation. Canadian Journal of Experimental Psychology, 54(2), 141-148.

Lemaire, P., \& Lecacheur, M. (2001). Older and younger adults' strategy use and execution in currency conversion tasks: Insights from French franc to euro and euro to French franc conversions. Journal of Experimental Psychology-Applied, 7(3), 195-206. 
La estimación en el cálculo y en la medida: fundamentación curricular e investigaciones desarrolladas en el Departamento de Didáctica de la Matemática de la Universidad de Granada

Lentzinger, Rathmell y Urbatsch (1986). Developing Estimating Skills in de Primary Grades. En H.L. Schoen \& M.J. Zweng (Eds.). Estimation and mental computation. Yearbook 82-92. N.C.T.M. Reston, VA.

Levin, J. (1981). Estimation techniques for arithmetic: everday math and mathematics instruction. Educational Studies in Mathematics, 12, 421-439.

Levine, D. R. (1980). Computational estimation ability and the use of estimation strategies among college students. Doctoral dissertation. New York University.

Levine, D. R. (1982). Strategy use and estimation ability of college students. Journal for Research in Mathematics Education, 15(5), 350-359.

Lynchard, B. B. (1989). The relationship of computational estimation ability and selected variables of sixth grade students. (Doctoral dissertation, Delta State University, 1988). Dissertation Abstracts International, 49A, 1686. (DA8814743).

McLaughin, J. (1981). Development of children's abilty to judge relative numerosity. Journal of experimental child psychology, 31, 103-114.

Markovits, Z. (1987)._Estimation. Research and curriculum development. Thesis submitted to the Scientific Council of the Weizmann Institute of Science Rehovot, Israel.

Markovits, Z. y Hershkowitz, R. (1993). Visual estimation of discrete quantities. ZDM. 93/4, 137-140.

Markovits, Z., \& Sowder, J. (1994). Developing number sense: An intervention study in grade 7. Journal for Research in Mathematics Education, 25(1), 4-29.

M. E. C. (1992). Propuestas de secuencia matemáticas. Editorial Escuela Española. S.A. Madrid. Montgomery, M. (1990). Results of the fourth NAEP mathematics assessment of the National Assessement of Educational Progress. NCTM. Reston VA.

Morgan, C. (1990). Factors affecting children's strategies and success in estimation. In G. Booker, P. Cobb, \& T. N. de Mendicuti (Eds.), Proceedings of the Fourteenth Psychology of Mathematics Education Conference (pp. 265-272). Mexico.

Mottran, R. D. (1996). A comparative study of computacional estimation ability and strategies used in estimation problems (Doctorar dissertation, University of Colorado at Boulder, 1995). Dissertation Abstracts International, 57/02A, 614. (DA9620647).

Murphy, M. R. (1992). Teaching and applyng computacional estimation skills in grade 8 . (Master's thesis, Simon Fraser University, Canada, 1989). Masters Abstracts Intenational, 30/04, 986. (DAMM66273).

N.C.T.M. (1989). Estandares curriculares y de evaluación para la educación matemática (SAEM THALES). Reston, VA. 
N. C. T. M. (1991). Estándares curriculares y de evaluación para la educación matemática. (SAEM THALES). Reston, VA.

Nieto, P. y otros. (1989). Diseño curricular de matemáticas. Enseñanza obligatoria 12-16. Consejería de Educación y Ciencia de la Junta de Andalucía. Sevilla.

Pareja, J. L. (2001). Estimación de cantidades discretas por alumnos de Magisterio (Memoria de Tercer Ciclo). Departamento de Didáctica de la Matemática de la Universidad de Granada. Piaget, J. y Szeminska, A. (1964). Génesis del número en el niño. Guadalupe. Buenos Aires.

Reehm, S. P. (1994). A comparison of estimation processes used on numeric and contextual problems. (Doctoral dissertation, University of Missouri-Columbia, 1992). Dissertation Abstracts International, 54/07, 2499A. (DA9400056).

Reys, R., Bestgen, B., Rybolt, J. y Wyatt, J. (1982). Processes used by good computational estimators. Journal for Research in Masthematics Education, 12(3), 183-201.

Reys, R. (1984). Mental computation and estimation: Past, present, and future. The Elementary School Journal, 84, 547-557.

Reys, B. J., Reys, R. E., \& Flores, A. (1991). Estimation performance and strategy use of Mexican 5th and 8th grade student sample. Educational Studies in Mathematics, 22(4), 353-375.

Reys, R. E. (1993). Research on computational estimation: What it tells us and some questions that need to be addressed. Hiroshima Journal of Mathematics Education, 1, 105-112.

Reys, R. E., Bestgen, B. J., Rybolt, J. F., \& Wyatt, J. W. (1982). Processes used by good computational estimators. Journal for Research in Mathematics Education, 12(3), 183201.

Reys, R. E., Bestgen, B. J., Trafton, P. R., \& Zawojewski, J. S. (1984). Computational estimation instructional materials for the middle grades. Final report. Washington, DC: National Science Foundation.

Reys, R. E., Reys, B. J., Nohda, N., Ishida, J., Yoshikawa, S., \& Shimizu, K. (1991). Computational estimation performance and strategies used by fifth and eighth-grade Japanese students. Journal for Research in Mathematics Education, 22(1), 39-58.

Rubenstein, R. (1985). Computational estimation and related mathematical skills. Journal for Research in Mathematics Education, 16(2), 106-119.

Sanfiorenzo, N. R. (1990). A comparison of teaching strategies in computational estimation. (Doctoral dissertation College for teachers of Vanderbilt University, 1989). Dissertation Abstracts International, 50/12, 3880A. (DA9006834). 
Schoen, H. L. (1994). Assessing computational estimation: Research and new directions. In R. E. Reys, \& N. Nohda (Eds.), Computational alternatives for the twenty-first century. Cross-cultural perspectives from Japan and the United States (pp. 87-97). Reston, Va: NCTM.

Schoen, H. L., Blume, G., \& Hart, E. (1987, April). Measuring computational estimation processes. Paper presented at the Annual Meeting of the American Educational Research Association. Washington, DC.

Schoen, H. L., Blume, G., \& Hoover, H. D. (1990). Outcomes and processes on estimation test items in different formats. Journal of Research in Mathematics Education, 21(1), $61-73$.

Schoen, H. L., Friesen, Ch. D., Jarrett, J. A., \& Urbatsch, T. D. (1981). Instruction in estimating solutions of whole number computations. Journal of Research in Mathematics Education, 12(3), 165-178.

Segovia, I. (1986). Estimación y cálculo aproximado en EGB. Tesina de licenciatura. Universidad de Granada. Departamento de Didáctica de la Matemática.

Segovia, I., Castro, E., Castro, E. y Rico, L. (1989). Estimación en cálculo y medida. Madrid: Síntesis.

Segovia, I. (1997). Estimación de cantidades discretas. Estudio de variables y procesos. Comares. Granada

Shumway, R. J. (1994). Some common directions for future research related to computational alternatives. In R. E. Reys, \& N. Nohda (Eds.), Computational alternatives for the twenty-first century. Cross-cultural perspectives from Japan and the United States (pp. 187-95). Reston, Va: NCTM.

Siegel, A. W., Goldsmith, L.T. y Madson, C. R. (1982). Skill in estimation problems of extent and numerosity. Journal for Research in Mathematics Education, 13(3), 211-232.

Sliva, W. M. (1988). Developing a test of computational estimation ability. (Doctoral dissertation University of Texas at Austin, 1987). Dissertation Abstracts International, 48A, 2567. (DA8728643).

Smith, M. L. (1993). Preservice elementary teachers' conceptual understanding of computational estimation strategies within the operations of addition and subtraction. (Doctoral dissertation, University of Pittsburgh, 1993). Dissertation Abstracts International, 54/06, 208A. (DA9329494).

Sowder, J. T. (1984). Computational estimation procedures of school children. Journal of Educational Research, 77(6), 332-36. 
Sowder, J. (1988). Mental computation and number comparison: their roles in the development of number sense and computational estimation. En J. Hiebert y M. Behr, (Eds). Number concepts and operations in the middle grades. Vol. 2. NCTM. Reston. Va.

Sowder, J. T. (1989). Affective factors and computational estimation ability. In D. B. McLeod, \& V. M. Adams (Eds.), Affect and mathematical problem solving: A new perspective (pp. 177-191). New York: Springer-Verlag.

Sowder, J. T. (1992). Estimation and number sense. In D. A. Grows (Ed.), Handbook of research on mathematics teaching and learning. A Project of the NCTM (pp. 371-389). New York: Macmillan Publishing Company.

Sowder, J. T. (1994). Cognitive and metacognitive processes in mental computation and computational estimation. In R. E. Reys, \& N. Nohda (Eds.), Computational alternatives for the twenty-first century. Cross-cultural perspectives from Japan and the United States (pp. 139-46). Reston, Va: NCTM.

Sowder, J. T., \& Markovits, Z. (1990). Relative and absolute error in computational estimation. In G. Booker, P. Cobb, \& T. N. de Mendicuti (Eds.), Proceedings of the Fourteenth Psychology of Mathematics Education Conference (pp. 321-328). Mexico.

Sowder, J. T., \& Wheeler, M. (1989). The development of concepts and strategies used in computational estimation. Journal for Research in Mathematics Education, 20(2), 130146.

Thompson, C. S. y Rathmell, E. C. (1989). By way of introduction. Arithmetic Teacher, 36(6), p.2.

Trafton, P. (1986). Teaching computational estimation: establishing an estimation mind set. En H.L. Schoen \& M.J. Zweng (Eds.). Estimation and mental computation (pp. 16-30). NCTM. Reston, VA.

Usiskin, Z. (1986). Reasons for estimating. En En H.L. Schoen \& M.J. Zweng (Eds.). Estimation and mental computation. NCTM. Reston, VA.

Whalen, M. T. (1989). A comparison of computer assited instruction to tradicional classroom instruction on seventh graders' computational estimation skills. (Doctoral dissertation, Indiana University, 1988). Dissertation Abstracts International, 49/12, $3650 \mathrm{~A}$. (DA8904969).

Whiteman, F. C. (1989). The role of computer-based instruction in the development of strategies for computational estimation with middle school children. (Doctoral dissertation, Ohio State University, 1988). Dissertation abstracts Insrtanional, 49/09, $2629 \mathrm{~A}$. (DA8824640). 
La estimación en el cálculo y en la medida: fundamentación curricular e investigaciones desarrolladas en el Departamento de Didáctica de la Matemática de la Universidad de Granada

Wyatt, J. W. (1985). A case-study survey of computational estimation processes and notions of reasobaleness amog ninth grade students. Doctoral dissertation. University of MissouryColumbia. 


\section{Anexo I . Test Segovia}

(El alumno debe elegir la mejor aproximación del resultado de cada operación, entre cinco opciones)

1. $32125+46164=(\quad)$

$\begin{array}{lllll}\text { (a) } 50000 & \text { (b) } 60000 & \text { (c) } 70000 & \text { (d) } 80000 & \text { (e) } 90000\end{array}$

2. $32125+40164=(\quad)$
(a) 65000
(b) 70000
(c) 75000
(d) 80000
(e) 85000

3. $52137+64215=(\quad)$
(a) 112000
(b) 114000
(c) 116000
(d) 118000
(e) 120000

4. $81215+92107=(\quad)$
(a) 160000
(b) 170000
(c) 180000
(d) 190000
(e) 200000

5. $39203+29107=(\quad)$
(a) 63000
(b) 65000
(c) 67000
(d) 69000
(e) 71000

6. $34107+57209=(\quad)$
(a) 80000
(b) 85000
(c) 90000
(d) 95000
(e) 100000

7. $59206+69105=(\quad)$
(a) 110000
(b) 120000
(c) 130000
(d) 140000
(e) 150000

8. $73107+68310=(\quad)$
(a) 135000
(b) 140000
(c) 145000
(d) 150000
(e) 155000

9. $37102+28015+16007=(\quad)$
(a) 75000
(b) 77000
(c) 79000
(d) 81000
(e) 83000

10. $19106+29001+16003=(\quad)$
(a) 60000
(b) 65000
(c) 70000
(d) 75000
(e) 80000

11. $62121+83003+93111=(\quad)$
(a) 233000
(b) 235000
(c) 237000
(d) 239000
(e) 241000

12. $60121+81321+91107=(\quad)$
(a) 200000
(b) 210000
(c) 220000
(d) 230000
(e) 240000

13. $76325-44103=(\quad)$ 

(a) 25000
(b) 30000
(c) 35000
(d) 40000
(e) 45000

14. $59763-21212=(\quad)$
(a) 31000
(b) 33000
(c) 35000
(d) 37000
(e) 39000

15. $71875-19621=(\quad)$
(a) 30000
(b) 40000
(c) 50000
(d) 60000
(e) 70000

16. $57645-39134=(\quad)$
(a) 15000
(b) 20000
(c) 25000
(d) 30000
(e) 35000

17. $84643-77132=()$
(a) 5000
(b) 6000
(c) 7000
(d) 8000
(e) 9000

18. $4018 \times 6=(\quad)$
(a) 23000
(b) 24000
(c) 25000
(d) 26000
(e) 27000

19. $4915 \times 4=()$

$\begin{array}{lllll}\text { (a) } 16000 & \text { (b) } 17000 & \text { (c) } 18000 & \text { (d) } 19000 & \text { (e) } 20000\end{array}$

20. $4213 \times 4=(\quad)$
(a) 15000
(b) 16000
(c) 17000
(d) 18000
(e) 19000

21. $4312 \times 7=(\quad)$
(a) 28000
(b) 29000
(c) 30000
(d) 31000
(e) 32000

22. $295 \times 406=(\quad)$
(a) 80000
(b) 90000
(c) 100000
(d) 110000
(e) 120000

23. $59347: 3=(\quad)$

$\begin{array}{lllll}\text { (a) } 10000 & \text { (b) } 20000 & \text { (c) } 30000 & \text { (d) } 40000 & \text { (e) } 50000\end{array}$

24. $34568: 7=(\quad)$
(a) 4000
(b) 5000
(c) 6000
(d) 7000
(e) 8000

25. $83745: 19=(\quad)$
(a) 5000
(b) 6000
(c) 7000
(d) 8000
(e) 9000

26. $42750: 19=(\quad)$
(a) 1000
(b) 2000
(c) 3000
(d) 4000
(e) 5000 
Isidoro Segovia et al.

27. $43000: 21=(\quad)$
(a) 5000
(b) 4000
(c) 3000
(d) 2000
(e) 1000 


\section{Anexo II}

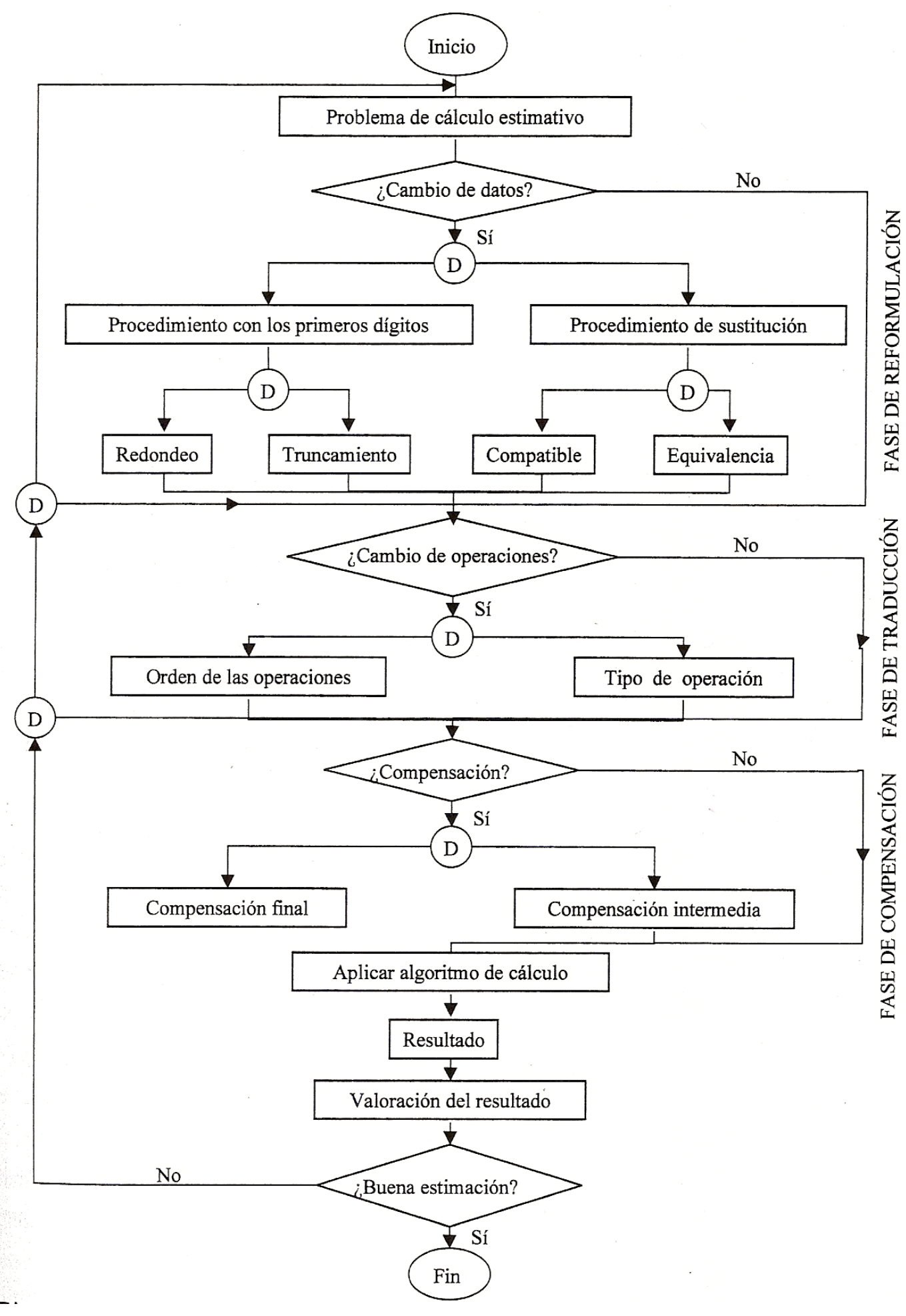




\section{Anexo III}

\section{Test de Levine}

$76 \times 89$

$93 \times 18$

$145 \times 37$

$824 \times 26$

$187,5 \times 0,06$

$482 \times 51,2$

$64,6 \times 0,16$

$424 \times 51,2$

$12,6 \times 11,4$

$0,47 \times 0,26$

$9208: 32$

$4645: 18$

$7858: 51$

$25410: 65$

$648,9: 22,4$

$546: 33,5$

$1292,8: 71,2$

$66: 0,86$

$943: 0,48$

$0,76: 0,89$ 